\title{
Chirality-Enhanced Periodic Self-Focusing of Light in Soft Birefringent Media
}

\author{
Guilhem Poy®, ${ }^{1, *}$ Andrew J. Hess, ${ }^{2}$ Ivan I. Smalyukh $\oplus^{2,3,4}$ and Slobodan Žumer $\odot^{5,1, \dagger}$ \\ ${ }^{1}$ Faculty of Mathematics and Physics, University of Ljubljana, Jadranska 19, 1000 Ljubljana, Slovenia \\ ${ }^{2}$ Department of Physics, 390 UCB, University of Colorado Boulder, Boulder, Colorado 80309, USA \\ ${ }^{3}$ Materials Science and Engineering, 027 UCB, Sustainability, Energy \& Environment Community, \\ University of Colorado Boulder, Boulder, Colorado 80303, USA \\ ${ }^{4}$ Renewable and Sustainable Energy Institute, National Renewable Energy Laboratory and University of Colorado, \\ Boulder, Colorado 80309, USA \\ ${ }^{5}$ Jožef Stefan Institute, Jamova cesta 39, 1000 Ljubljana, Slovenia
}

(Received 15 May 2020; accepted 10 July 2020; published 11 August 2020)

\begin{abstract}
We experimentally and numerically show that chirality can play a major role in the nonlinear optical response of soft birefringent materials, by studying the nonlinear propagation of laser beams in frustrated cholesteric liquid crystal samples. Such beams exhibit a periodic nonlinear response associated with a bouncing pattern for the optical fields, as well as a self-focusing effect enhanced by the chirality of the birefringent material. Our results open new possible designs of nonlinear optical devices with low power consumption and tunable interactions with localized topological solitons.
\end{abstract}

DOI: 10.1103/PhysRevLett.125.077801

Solitons are fascinating realizations of self-reinforcing wave solutions that generally rely on nonlinear couplings to compensate dispersive effects. Solitons emerge in a range of systems including water canals [1], Bose-Einstein condensates [2], and nerve pulse propagation in biological membranes [3], and play a major role in the field of photonics as effective modes of the flow of light in isotropic [4], birefringent [5], and metamaterial [6] systems. In some systems, chirality - the breaking of mirror symmetry-is essential in the emergence of new classes of propagating nonlinear waves. For example, the chiral edge states of the quantum Hall effect are closely linked to solitonic solutions of the chiral nonlinear Schrödinger equation $[7,8]$, and complex vector solitons appear in chiral media with Kerrtype nonlinear optical response through the Born-Fedorov mechanism $[9,10]$.

Interestingly, the relationship between chirality and effective modes of wave propagation has attracted an interest beyond solitonic science. For example, the existence of chiral light-matter interactions with slow light was demonstrated in glide-plane-symmetric photonic waveguides [11]; a giant chiral optical response was achieved with twisted-arc metamaterials [12]; and nonreciprocal propagation was realized with flying couplers and spinning resonators [13].

Published by the American Physical Society under the terms of the Creative Commons Attribution 4.0 International license. Further distribution of this work must maintain attribution to the author(s) and the published article's title, journal citation, and DOI.
Chirality also plays a fundamental role in the elastic orientational response of cholesteric liquid crystal (CLC) a chiral phase with orientational order-with the appearance of many metastable states and the stabilization of knotted orientational fields in confined geometries [14-16]. Since the nonlocal elastic reorientation of molecules under the action of an external optical field is known to give rise to nonlinear optical effects and spatial optical solitons in achiral liquid crystal [5,17]—dubbed nematicons - the following important question arises: How does the intrinsic chirality of a CLC affect the nonlinear optical response of this birefringent material? Although previous experiments and simulations showed the existence of optical solitons in CLC with the usual cholesteric helix texture [18-20], this question was never answered since these works focused on bistability, waveguiding and elastic anisotropy and not on the optical nonlinearity itself.

In this Letter, we address this question and show that chirality can enhance the optical nonlinearity of CLC, thus paving the way for nonlinear photonic device at low optical power. With experimental and theoretical approaches, we disclose the self-focusing mechanism of optical beams propagating in confinement-induced unwound CLC with a periodic nonlinear optical response which, to the best of our knowledge, was not observed before.

Contrary to previous observation of solitons in CLC [18-20], we focus here on frustrated CLC (FCLC) in which the equilibrium state of the director field $\boldsymbol{n}$ is not the well-known cholesteric helix-which is associated with a periodicity length $p$, called the CLC pitch-but the much simpler unwound uniform state $\boldsymbol{n}_{0}$. This was experimentally achieved through the use of strong anchoring 


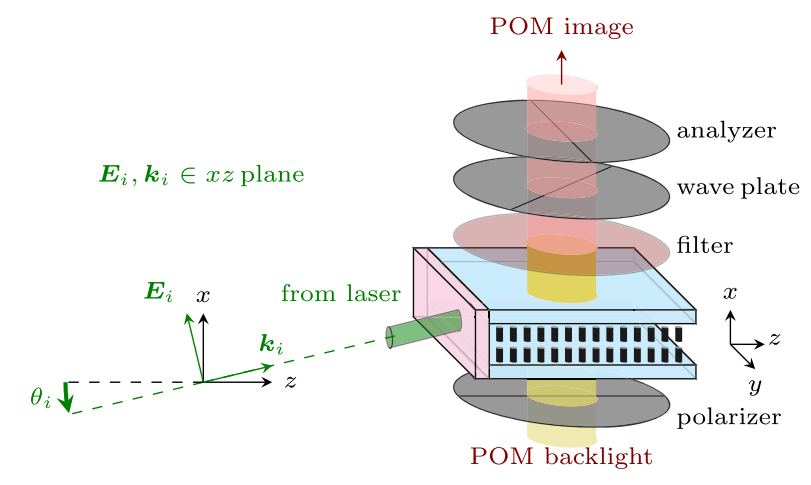

FIG. 1. Schematic of the experimental setup. The CLC layer is sandwiched between two plates treated for homeotropic boundary conditions, and the laser beam is injected from the side through a cover slip (in pink) also treated for vertical alignment of the CLC molecules. For sufficiently thin samples, the equilibrium bulk orientation of the CLC molecules is the unwound state $\boldsymbol{n}_{0}$ parallel to $x$.

treatment on the confining glass plates of the sample imposing a vertical alignment of the CLC molecules $\left(\boldsymbol{n}_{0} \equiv \boldsymbol{e}_{x}\right)$, as depicted in Fig. 1. We worked with $17 \mu \mathrm{m}$ or $30 \mu \mathrm{m}$-thick CLC samples associated with a thicknessto-pitch ratio $h / p$ of $0.8-0.9$, which ensured $\boldsymbol{n}=\boldsymbol{n}_{0}$ when no light was sent into the sample. An objective with $\mathrm{NA}>0.50$ collimated a $532 \mathrm{~nm}$ laser beam into the cell, at powers $<1 \mathrm{~mW}$ in the linear optical regime [21] and between $20-40 \mathrm{~mW}$ in the nonlinear regime. The sample was examined with a polarized optical microscope (POM). With the broadband white backlight on in Fig. 1, the reorientation of CLC molecules can be directly observed using a removable polarizer, analyzer, and wave plate with a red-pass filter to absorb the green scattered light from the laser; conversely, if the POM backlight is off, the laser intensity can be directly seen in the microscope thanks to light scattering by the CLC orientational fluctuations.

In our simulations, we coupled a wide-angle beampropagation method [22] with a standard minimization scheme of the CLC free energy assuming a constant scalar order parameter [23,24], thus allowing to simultaneously calculate the optical and director fields in the CLC layer and simulate POM images. The nonlinear optical response results from the reorientation of CLC molecules-initially aligned with $x$-due to the electric torque imposed by the optical fields (represented schematically as $\boldsymbol{E}_{i}$ on Fig. 1 for the input beam), thus inducing a change in the anisotropic permittivity tensor and therefore the effective refractive index seen by the optical beam. Note that the input beam needs to be tilted in the $x z$ plane in order to achieve a nonzero reorientation. All our simulations were performed in $10 \mu$ m-thick samples with a ratio $h / p=0.8$ (except for Fig. 4 where the pitch was changed). Details of the sample preparation, beam-propagation scheme and parameters are given in the Supplemental Material [25] (SM).

In Figs. 2(a)-2(d), we show experimental and numerical images of a beam propagating in the sample of Fig. 1 with an input angle $\theta_{i} \approx 13^{\circ}$. At low optical power [Figs. 2(a) and 2(c)], the beam diffracts linearly since the reorientation of the CLC molecules is negligible, while at higher power [Figs. 2(b) and 2(d)] the nonlinear optical response of the CLC can compensate the diffractive effects, with the shape of the transverse intensity profile [Fig. 2(e)] preserved across $\sim 0.4 \mathrm{~mm}(\sim 0.8 \mathrm{~mm})$ for the experiment (simulation) - both distances corresponding to the length between $\mathcal{L}_{1}$ and $\mathcal{L}_{3}$ in Figs. 2(b) and 2(d).

Figures 2(b), 2(d), and 2(e) present clear evidence of optical self-focusing, which in liquid crystals is usually due to molecular reorientation. As shown in Figs. 2(f)-2(h), the

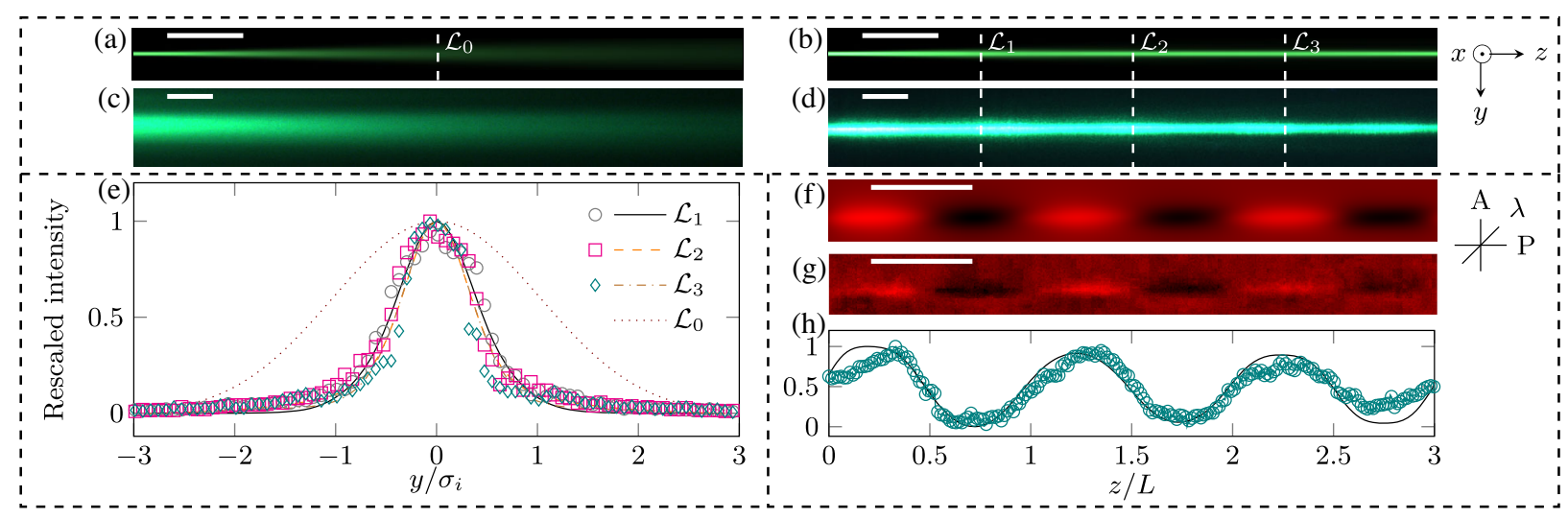

FIG. 2. Simulated (a),(b) ( $x$-averaged beam intensity) and experimental (c),(d) images (scattered laser light) of a beam propagating in a FCLC sample in the linear (a),(c) and nonlinear (b),(d) optical regime. Simulated (f) and experimental (g) POM images, with the orientations of the polarizer, $\lambda$-wave plate and analyzer represented schematically on the right. The white bars represent $100 \mu \mathrm{m}$ in (a)-(d) and the half-periodicity $L / 2$ of the POM signal in (f),(g) [43 $\mu \mathrm{m}$ for (f), $143 \mu \mathrm{m}$ for (g)]. The axes orientation on the right applies to (a)-(g). (e) Experimental (marks) and simulated (lines) rescaled intensity profiles along the lines $\mathcal{L}_{1,2,3}$ defined in (b),(d). $\sigma_{i}$ is the input beam waist. For comparison, the profile $\mathcal{L}_{0}$ of the diffracting beam presented in (a) is also shown. (h) Experimental (marks) and simulated (line) rescaled 1D profile of POM images (f), (g) along the beam axis. 


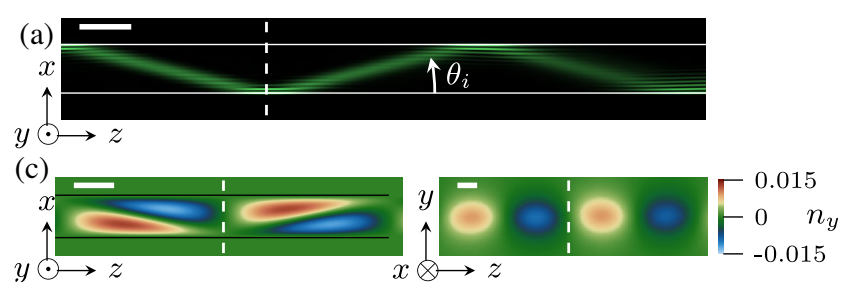

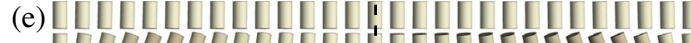

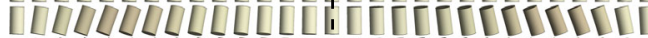

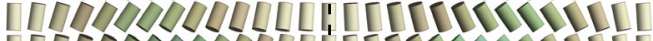

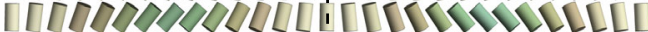

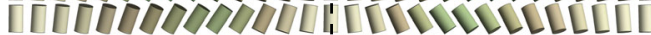

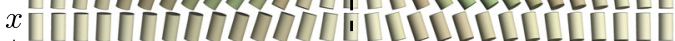

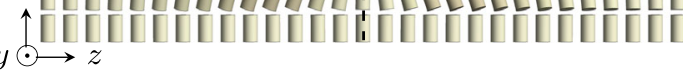

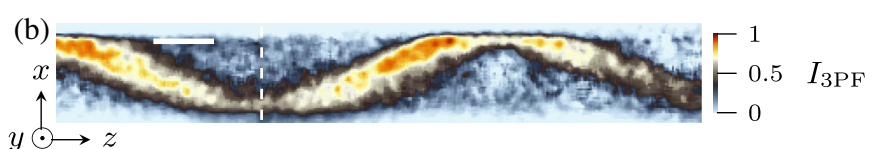

(d)

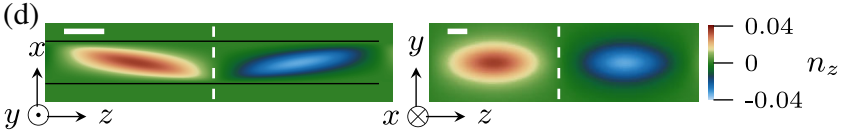

(f) 000000000000000000000000

f) (2909cos (0)

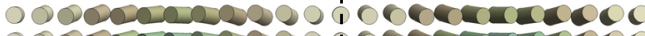

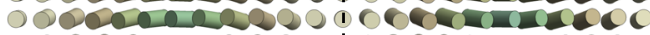

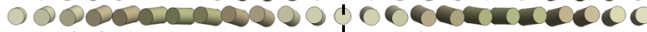
$y 000000000000,0000000000$

y

$\uparrow$ 个 $x \otimes{ }^{\circ} \rightarrow 000000000000000000000$

FIG. 3. $x z$ slice of the simulated beam intensity (a) and experimental tomography signal $I_{3 \mathrm{PF}}$ (b). (c) Color-coded slices of $n_{y}$ ( $y$ component of the director field) in the $x z$ and $y z$ planes. (d) Same as (c) with $n_{z}$. (e),(f) Schematic representation of the reorientation of the director (depicted with cylinders) in the $x z$ and $y z$ planes. The reorientation angle was greatly exaggerated and the $\{x, y\}$ directions were magnified for clarity. The white bars represent $10 \mu \mathrm{m}$. The dashed lines represent the same $z$.

experimental and simulated POM images-whose contrast is related to the reorientation of CLC molecules-are periodic along the beam axis, in stark contrast to fundamental optical solitons in achiral liquid crystals [17]. When the beam axis is parallel to the polarizer [Figs. 2(f) and $2(\mathrm{~g})]$, the POM signal along this axis corresponds to a sinelike variation with dark and bright spots, also visible in Fig. 2(h) where rescaled 1D profiles of Figs. 2(f) and 2(g) along the beam axis are plotted. As a general rule, the POM signal depends on many parameters (see SM for additional data)_including sample thickness, beam power, insertion angle, and polarizer orientation-but always has a welldefined periodicity length $L$ which scales linearly with the sample thickness, both in experiments and numerical simulations.

To determine the physical origin of this periodicity, we plotted in Fig. 3(a) a $x z$ slice of the simulated beam intensity. As visible, total internal reflection occurs at the interfaces between the CLC layer and the glass plates, and the beam is in fact bouncing between the two plates and stays confined inside the CLC layer despite the nonzero insertion angle. The periodicity $L$ associated with this bouncing is identical to the periodicity of the POM signal in Fig. 2 and can be solely determined from the sample thickness $h$ and insertion angle $\theta_{i}$ (see Fig. 1) with the relation $L=2 h / \tan \theta_{i}$. Going back to Fig. 2(f), this relation indeed predicts the correct periodicity of $\sim 86 \mu \mathrm{m}$ since in the simulations the sample thickness was $10 \mu \mathrm{m}$ and the insertion angle was $13^{\circ}$. We also mention that this bouncing pattern relies on self-focusing in the $x$ direction and was not observed in the linear optical regime (see SM).

To confirm that the same explanation also applies in the experiments, we filled a $16.9 \mu$ m-thick sample with a CLC mixture associated with a ratio $h / p \approx 0.8-1$ and doped with photopolymerizable molecules (composition given in the SM). While the laser beam propagated through the
FCLC layer, the sample was exposed to a weak UV source to initiate photopolymerization and directly imaged using label-free three-photon-fluorescence tomographic microscopy with $80 \mathrm{~mW}$ incident power as described by Lee et al. [36]. Tomography micrographs revealed a tubelike structure that bounced between its confining glass plates as shown with a $x z$ slice in Fig. 3(b) and with additional slices in the SM. The intensity profile $I_{3 \mathrm{PF}}$ in Fig. $3(\mathrm{~b})$ and in the SM is defined as the observed tomography signal $I_{\text {observed }}$ subtracted from the background $I_{\text {background }}$. The tomography intensity contrast is due to partial self-polymerization around the laser beam prior to full photopolymerization, thereby causing a partial exclusion of CLC molecules from the tube. This process is similar to impurity exclusion for systems with two coexisting thermodynamic phases [37]. The tubelike structure indicates that simulations such as the one in Fig. 3(a) accurately describe the self-localized beam's bouncing behavior in the experiment.

In addition, Fig. 3 also provides color-coded slices of the director field components in the $x z$ plane and $y z$ plane, as well as a schematic representation of the director reorientation with cylinders. Most importantly, the reorientation of CLC molecules is not purely contained in the $x z$ plane (as it should be in an achiral sample with a beam polarization in the $x z$ plane), but is fully three dimensional. This symmetry breaking happens because of the natural tendency of CLC to form twisted configurations; as visible in Figs. 3(e) and 3(f), twisted domains locally appear and on average have the same handedness as the intrinsic chirality.

We now come back to the main question of this Letter concerning the relation between chirality and nonlinear optical response. For negligible optical power, the permittivity tensor is simply $\boldsymbol{\epsilon}^{(\boldsymbol{\theta})}=n_{e}^{2} \boldsymbol{n}_{0} \otimes \boldsymbol{n}_{0}+n_{o}^{2}\left(\mathbf{I}-\boldsymbol{n}_{0} \otimes\right.$ $\left.\boldsymbol{n}_{0}\right)$ in the LC layer and $\boldsymbol{\epsilon}^{(\boldsymbol{\theta})}=n_{p}^{2} \mathbf{I}$ in the confining plates, where $\boldsymbol{n}_{0} \equiv \boldsymbol{e}_{x}$ is the equilibrium value of the director field 
in the FCLC layer and $n_{e, o, p}$ are the extraordinary, ordinary, and confining plates' refractive indices. To first order in the reorientation angle, the permittivity tensor in the nonlinear regime is written as $\boldsymbol{\epsilon}=\boldsymbol{\epsilon}^{(\boldsymbol{\theta})}+\boldsymbol{\delta} \boldsymbol{\epsilon}$ with $\boldsymbol{\delta} \boldsymbol{\epsilon} \equiv\left(n_{e}^{2}-n_{o}^{2}\right) \times$ $\left(\boldsymbol{n}_{0} \otimes \boldsymbol{n}_{\perp}+\boldsymbol{n}_{\perp} \otimes \boldsymbol{n}_{0}\right)$ and $\boldsymbol{n}_{\perp}=\left\{0, n_{y}, n_{z}\right\}$. Based on the formalism of Refs. [22,37], neglecting optical activity and keeping only nonlinear terms of the lowest order, we then find that the director and optical fields can be calculated from the following system of differential equations:

$$
\begin{gathered}
\Delta \boldsymbol{n}_{\perp}+2 q \frac{\partial \boldsymbol{n}_{\perp}}{\partial x} \times \boldsymbol{n}_{0}=-\frac{\epsilon_{0} n_{o}^{2} \alpha}{4 K} \operatorname{Re}\left(\left[\boldsymbol{E} \cdot \boldsymbol{n}_{0}\right]^{*} \boldsymbol{E}\right), \\
\frac{\partial E_{x}}{\partial z}=i\left[\sqrt{k_{0}^{2} \epsilon_{x x}^{(0)}+D^{(0)}}+i \alpha n_{z} \frac{\partial}{\partial x}\right] E_{x}, \\
E_{z}=\left[k_{0}^{2} \epsilon_{z z}^{(0)}+\Delta\right]^{-1}\left[\frac{\partial^{2}}{\partial z \partial x}-\alpha n_{o}^{2} n_{z}\right] E_{x},
\end{gathered}
$$

with $\alpha \equiv\left(n_{e} / n_{o}\right)^{2}-1, q \equiv 2 \pi / p$ the spontaneous twist of the cholesteric phase, $K$ the Frank elastic constant (assuming isotropic elasticity), $k_{0}$ the wave vector in empty space, and $D^{(0)}$ a diffraction operator containing second order derivatives in $x$ and $y$ :

$$
\forall A, \quad D^{(0)} A \equiv \frac{\partial}{\partial x}\left(\frac{1}{\epsilon_{z z}^{(0)}} \frac{\partial}{\partial x}\left[\epsilon_{x x}^{(0)} A\right]\right)+\frac{\partial^{2} A}{\partial y^{2}} .
$$

Note that in Eqs. (1)-(3), we neglected the $y$ component of the optical field $\boldsymbol{E}$. This assumption can be justified empirically from our numerical simulations, in which we systematically found that $E_{y} \ll E_{x, z}$ when the initial polarization is in the $x z$ plane, or theoretically from Maxwell's equations, which give the estimation $E_{y}=$ $\mathcal{O}\left[\alpha n_{y} E_{x},\left(\partial^{2} E_{x} / \partial x \partial y\right) /\left(n_{o} k_{0}\right)^{2}\right]$ for our system.

Equation (1) allows the calculation of the director field $\boldsymbol{n}$ for a given optical field $\boldsymbol{E}$, whereas Eqs. (2) and (3) allow the calculation of $\boldsymbol{E}$ for a given $\boldsymbol{n}$ and fully include the jump of permittivity at the interfaces between the FCLC layer and the glass plates. For an achiral sample, $q=0$ and Eq. (1) shows that the director field stays in the same plane formed by $\boldsymbol{E}$ and $\boldsymbol{n}_{\boldsymbol{0}}$. Conversely, if $q \neq 0$ as in this Letter, the director field reorientation is fully $3 \mathrm{D}$ with a nonzero $n_{y}$, as shown previously in Fig. 3. At low optical power, $n_{z} \approx 0$ and Eq. (2) show that the optical fields must diffract due to the action of the operator $D^{(0)}$. For higher optical power, $n_{z}$ becomes periodically nonzero around the beam and allows the compensation of the diffraction in Eq. (2), thus producing periodic self-focusing of the beam. In the SM, we show how Eq. (2) can be directly compared with common propagation models of solitons in achiral liquid crystal and give a few additional theoretical results, while we focus here on the general physics behind Eqs. (2) and (3).

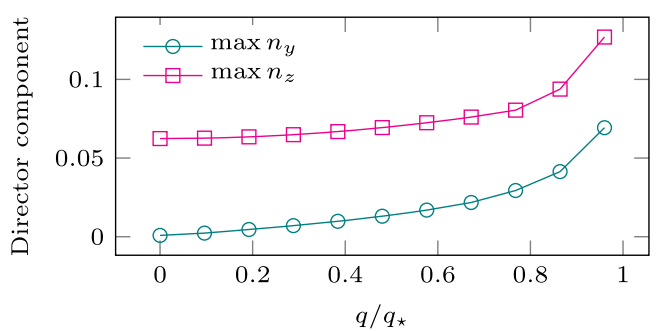

FIG. 4. Maxima of $n_{y}$ and $n_{z}$ plotted as a function of $q / q_{\star}$, assuming that all other simulation parameters (beam power, insertion angle, sample thickness) stay constant.

From the discussion above, we conclude that, in our system, a nonzero $n_{y}$ is the visible trace of chirality while a nonzero $n_{z}$ induces self-focusing effects in Eq. (2). To estimate how chirality affects the amplitude of molecular reorientation-and therefore of the self-focusing-we performed 11 simulations similar to the one presented in Figs. 2 and 3, each time keeping the same beam power, insertion angle, and sample thickness but changing the spontaneous twist $q$ from 0 to slightly below the threshold of destabilization $q_{\star} \approx \pi / h$ of the unwound state in the FCLC layer [37]. The main result of these simulations is shown in Fig. 4, where the maxima of $n_{y}$ and $n_{z}$ in the whole computational box are plotted as a function of $q / q_{\star}$. As expected from Eq. (1), $\max n_{y}$ is zero when $q=0$ and increase with $q$ in chiral samples. More interestingly, $n_{z}$-which is nonzero in achiral sample-also increases with the spontaneous twist, with an enhancement factor of $\sim 2$ just below $q_{\star}$. This enhancement effect was also observed in the experiments, with the raw POM intensity contrast along the beam axis multiplied by $\sim 1.7$ when increasing $h / p$ from 0.6 to 0.8 .

Figure 4 therefore provides a clear answer to our initial question: chirality is indeed very much relevant to the nonlinear optical response of birefringent media and can be leveraged to enhance self-focusing effects in FCLC systems. This effect can be understood as follows: the equilibrium state in FCLC is highly frustrated because the director field is initially untwisted, but the laser beam allows local destabilization of this unwound state, thus creating locally twisted structures [as seen in Figs. 3(e) and $3(\mathrm{f})$ ] and partially relieving frustration. We deduce that in CLC both the optical fields and the spontaneous twist are responsible for the director field reorientation, whereas in usual nematic liquid crystal, only the optical fields induce a reorientation. Our explanation is reinforced by the fact that $n_{y}$ and $n_{z}$ sharply increase as $q \rightarrow q_{\star}$, which can be interpreted as a local shifting and softening of the transition from unwound to twisted CLC state.

Up until now, we carefully avoided using the term optical soliton for the self-focused optical waves of Figs. 2 and 3, since the nonconventional bouncing pattern of the beam and the chirality enhancement effect are not present in the usual case of fundamental solitons with a 
translationally invariant nonlinear response [17]. However, we emphasize that translational invariance is not a requirement for spatial solitons; for example, periodic modulation of the beam intensity are present in the breathing modes of nematicons [17]. For this reason, we believe that the nonlinear optical waves of this Letter can reasonably be addressed as spatial solitons-since they exhibit selffocusing of the transverse profile-or even better as similaritons, a special case of spatial solitons in nonlinear media with inhomogeneous gain, diffraction and nonlinear response along the beam axis [38]. Note that bouncing solitons similar to Fig. 3 should also be observable in nematics since Fig. 4 shows that $n_{z}$ is nonzero even when $q=0$.

To summarize, this Letter provides evidence that chirality can play a major role in the nonlinear response of birefringent materials. We focused on FCLC systems and showed that self-focused nonlinear optical waves can be obtained with a striking bouncing pattern between the confining glass plates of the sample. Moreover, we showed that chirality directly contributes to the selffocusing effect, with an enhancement factor increasing sharply near the threshold of destabilization of the FCLC. Our results thus provide the basis for possible designs of nonlinear photonic devices where chirality actually helps to decrease the device's nominal optical power, e.g., for nonlinear flat lenses. Since FCLCs support the existence of birefringent topological solitons-topologically protected localized directorfield configurations [16]—our results also suggest novel breeds of all-optical information systems based on optical interaction between nonlinear waves and topological solitons.

G.P. and S. Ž. acknowledge funding from the ARSS (Javna Agencija za Raziskovalno Dejavnost RS) through Grant No. P1-0099 and from the European Unions Horizon 2020 program through the Marie Skłodowska-Curie Grant Agreement No. 834256 and COST action No. CA17139 (EUTOPIA). A. J. H. and I. I. S. acknowledge funding from the National Science Foundation through Grant No. DMR1810513. A. J.H. thanks the United States Office of Science Graduate Student Research (SCGSR) Fellowship for partial project support and the United States National Renewable Energy Laboratory's Technology Transfer Office for remuneration from the Technology Licensing and Commercialization Fellowship.

* Corresponding author.

guilhem.poy@fmf.uni-lj.si

${ }^{\dagger}$ Corresponding author.

slobodan.zumer@fmf.uni-lj.si

[1] D. J. Korteweg and G. De Vries, London Edinburgh Dublin Philos. Mag. J. Sci. 39, 422 (1895).
[2] C. Becker, S. Stellmer, P. Soltan-Panahi, S. Dörscher, M. Baumert, E.-M. Richter, J. Kronjäger, K. Bongs, and K. Sengstock, Nat. Phys. 4, 496 (2008).

[3] S. S. Andersen, A. D. Jackson, and T. Heimburg, Prog. Neurobiol. 88, 104 (2009).

[4] T. G. Philbin, C. Kuklewicz, S. Robertson, S. Hill, F. König, and U. Leonhardt, Science 319, 1367 (2008).

[5] M. Peccianti, A. Dyadyusha, M. Kaczmarek, and G. Assanto, Nat. Phys. 2, 737 (2006).

[6] N. N. Rosanov, N. V. Vysotina, A. N. Shatsev, A. S. Desyatnikov, and Y.S. Kivshar, Phys. Rev. Lett. 108, 133902 (2012).

[7] J.-H. Lee and C.-K. Lin, and O. K. Pashaev, Chaos Solitons Fractals 19, 109 (2004).

[8] H. Bulut, T. A. Sulaiman, and B. Demirdag, Nonlinear Dyn. 91, 1985 (2018).

[9] K. Hayata and M. Koshiba, IEEE Trans. Microwave Theory Tech. 43, 1814 (1995).

[10] D. D. Estelle Temgoua and T. C. Kofane, Phys. Rev. E 93, 062223 (2016).

[11] S. Mahmoodian, K. Prindal-Nielsen, I. Söllner, S. Stobbe, and P. Lodahl, Opt. Mater. Express 7, 43 (2017).

[12] Y. Cui, L. Kang, S. Lan, S. Rodrigues, and W. Cai, Nano Lett. 14, 1021 (2014).

[13] S. Maayani, R. Dahan, Y. Kligerman, E. Moses, A. U. Hassan, H. Jing, F. Nori, D. N. Christodoulides, and T. Carmon, Nature (London) 558, 569 (2018).

[14] U. Tkalec, M. Ravnik, S. Čopar, S. Žumer, and I. Muševič, Science 333, 62 (2011).

[15] D. Seč, S. Čopar, and S. Žumer, Nat. Commun. 5, 3057 (2014).

[16] P. J. Ackerman and I. I. Smalyukh, Phys. Rev. X 7, 011006 (2017).

[17] Nematicons: Spatial Optical Solitons in Nematic Liquid Crystals, edited by G. Assanto, Wiley Series in Pure and Applied Optics (John Wiley \& Sons, Hoboken, NJ, 2013).

[18] U. A. Laudyn, M. Kwasny, and M. A. Karpierz, Appl. Phys. Lett. 94, 091110 (2009).

[19] F. A. Sala and M. A. Karpierz, Opt. Express 20, 13923 (2012).

[20] A. Madani, J. Beeckman, and K. Neyts, Opt. Commun. 298-299, 222 (2013).

[21] The linear optical regime is defined here as the range of powers producing a CLC reorientation too weak to induce visible self-focusing effects.

[22] G. Poy and S. Žumer, Opt. Express 28, 24327 (2020).

[23] M. Ravnik and S. Žumer, Liq. Cryst. 36, 1201 (2009).

[24] G. Poy, F. Bunel, and P. Oswald, Phys. Rev. E 96, 012705 (2017).

[25] See Supplemental Material at http://link.aps.org/ supplemental/10.1103/PhysRevLett.125.077801 for additional experimental, numerical, and theoretical details, which includes Refs. [26-35].

[26] J. Cheng and R. B. Meyer, Phys. Rev. A 9, 2744 (1974).

[27] H. L. Ong, M. Schadt, and I. F. Chang, Mol. Cryst. Liq. Cryst. 132, 45 (1986).

[28] P. D. Brimicombe, C. Kischka, S. J. Elston, and E. P. Raynes, J. Appl. Phys. 101, 043108 (2007).

[29] H. N. Ritland, J. Am. Ceram. Soc. 38, 86 (1955).

[30] G. Poy and S. Žumer, Soft Matter 15, 3659 (2019). 
[31] M. A. Karpierz, Phys. Rev. E 66, 036603 (2002).

[32] C. P. Jisha, A. Alberucci, J. Beeckman, and S. Nolte, Phys. Rev. X 9, 021051 (2019).

[33] M. Sluijter, D. K. G. de Boer, and J. J. M. Braat, J. Opt. Soc. Am. A 25, 1260 (2008).

[34] C. Hu and J. R. Whinnery, J. Opt. Soc. Am. 64, 1424 (1974).

[35] C. Conti, M. Peccianti, and G. Assanto, Phys. Rev. E 72, 066614 (2005).
[36] T. Lee, R. P. Trivedi, and I. I. Smalyukh, Opt. Lett. 35, 3447 (2010).

[37] P. Oswald and P. Pieranski, Nematic and Cholesteric Liquid Crystals: Concepts and Physical Properties Illustrated by Experiments (CRC Press, Boca Raton, FL, 2006).

[38] S. A. Ponomarenko and G. P. Agrawal, Opt. Lett. 32, 1659 (2007). 